\title{
DIFUSÃO DA INOVAÇÃO TECNOLÓGICA e-SUS AB: ACEITAÇÃO OU REJEIÇÃO?*
}

\author{
Talita Ingrid Magalhães Silva1', Ricardo Bezerra Cavalcante², Hugo Ricardo Moreira da Silva ${ }^{3}$, Regina \\ Consolação Santos ${ }^{4}$, Eliete Albano de Azevedo Guimarães ${ }^{5}$, Marta Macedo Kerr Pinheiro ${ }^{6}$
}

\begin{abstract}
Objetivo: analisar a difusão da inovação tecnológica "estratégia e-SUS AB" em uma equipe de saúde da família do oeste de Minas Gerais. Método: estudo de caso único de abordagem qualitativa à luz da Teoria da Difusão da Inovação e do Modelo de Aceitação de Tecnologia. A coleta de dados compreendeu entrevista e observação direta do cotidiano de trabalho de uma equipe. Os dados foram sistematizados no software Atlas ti e analisados através da Análise de Conteúdo. Resultados: a inovação proporciona suporte no planejamento da assistência por dispor de dados individualizados, além de aprimorar o cotidiano de trabalho. Entretanto, sua difusão tem gerado, predominantemente, sobrecarga de trabalho e outras dificuldades que se conformam como limitadores de sua aceitação pelos profissionais de saúde. Conclusão: a difusão da estratégia e-SUS AB como uma inovação tecnológica tem apresentado situações potencializadoras de sua aceitação, porém predominaram situações que podem conduzir à sua rejeição.
\end{abstract}

DESCRITORES: Sistema de informação em saúde; Difusão de inovações; Informática em saúde; Tecnologia da informação; Atenção primária em saúde.

\section{DIFFUSION OF THE TECHNOLOGICAL INNOVATION e-SUS AB: ACCEPTANCE OR REJECTION?*}

Objective: analyze the diffusion of the technological innovation "e-SUS AB strategy" in a Family health team in the West of Minas Gerais. Method: single case study with a qualitative approach in the light of the Innovation Diffusion Theory and the Technology Acceptance Model. The data collection included an interview and direct observation of a team's daily work. The data were systemized in the software Atlas ti and analyzed through Content Analysis. Results: the innovation supports the care planning because it makes individualized data available and improves the daily work. Nevertheless, its diffusion has predominantly produced a work overload and other difficulties that limit its acceptance by the health professionals Conclusion: the diffusion of the e-SUS AB strategy as a technological innovation has presented situations that strengthen its acceptance, but situations that can lead to its rejection were predominant.

DESCRIPTORS: Health information systems; Diffusion of innovation; Medical informatics; Information technology; Primary health care.

\section{DIFUSIÓN DE LA INNOVACIÓN TECNOLÓGICA e-SUS AB: ACEPTACIÓN O RECHAZO?*}

Objetivo: analizar la difusión de la innovación tecnológica "estrategia e-SUS AB" en un equipo de salud de la familia del oeste de Minas Gerais. Método: estudio de caso único con enfoque cualitativo a la luz de la Teoría de Difusión de Innovaciones y del Modelo de Aceptación de Tecnología. Para recolectar los datos se llevaron a cabo entrevista y observación directa del trabajo cotidiano de un equipo. Los datos fueron sistematizados en el software Atlas ti y analizados a través del Análisis de Contenido. Resultados: la innovación provee apoyo en la planificación de la atención por disponer de datos individualizados, además de perfeccionar el trabajo cotidiano. Sin embargo, su difusión ha generado predominantemente sobrecarga de trabajo y otras dificultades que representan limitadores de su aceptación por los profesionales de salud. Conclusión: la difusión de la estrategia e-SUS AB como una innovación tecnológica ha presentado situaciones que potencializan su aceptación, pero predominaron situaciones que pueden llevar a su rechazo.

DESCRIPTORES: Sistemas de información en salud; Difusión de innovaciones; Informática médica; Tecnología de la información; Atención primaria de salud.

*Artigo extraído da Dissertação intitulada Difusão da inovação e-SUS atenção básica (e-SUS AB) em equipes de saúde da família. Universidade Federal de São João Del Rei, 2017.

${ }^{1}$ Enfermeira. Doutoranda de Enfermagem da Universidade Federal de Minas Gerais. Docente da Universidade do Estado de Minas Gerais. Belo Horizonte, MG, Brasil.

²Enfermeiro. Pós-doutorado em Ciência da Informação. Docente da Universidade Federal de São João Del Rei. Divinópolis, MG, Brasil.

${ }^{3}$ Discente de Enfermagem. Bolsista da FAPEMIG/UFSJ. Universidade Federal de São João Del Rei. Divinópolis, MG, Brasil.

${ }^{4}$ Enfermeira. Mestre em Ciências. Docente da Universidade Federal de São João Del Rei. Divinópolis, MG, Brasil.

${ }^{5}$ Enfermeira. Pós doutorado em Ciências da Saúde. Docente da Universidade Federal de São João Del Rei. Divinópolis, MG, Brasil. ${ }^{6}$ Pesquisadora. Pós-doutorado em Ciência da Informação e da Comunicação pela Universidade Paul Sabatier/IUT/Toulouse III. Docente da Universidade FUMEC e Professora Colaboradora do Programa de pós-graduação em Ciência da Informação da Universidade Federal de Minas Gerais. Belo Horizonte, MG, Brasil. 


\section{INTRODUÇÃO}

No Brasil, o reconhecimento da importância da informação para os processos decisórios tem fomentado o desenvolvimento e a implantação de diversos sistemas de informação em saúde.(1) Tais sistemas constituem a junção de componentes que instrumentalizam o processo de coleta, processamento, análise e disseminação da informação, potencializando a gestão da informação nos serviços de saúde. (1) Entretanto, por quase duas décadas, o Sistema de Informação da Atenção Básica (SIAB) foi utilizado para organizar e nortear o fluxo de informações e otimizar a gestão da informação da Atenção Básica à Saúde no Brasil. (2)

O Ministério da Saúde (MS), através do Departamento da Atenção Básica (DAB), buscando alternativa inovadora, propôs, em 2011, a estratégia e-SUS Atenção Básica (e-SUS AB). Com esta estratégia buscase a informatização qualificada do SUS, também denominada de SUS eletrônico. ${ }^{(3)} \mathrm{A}$ estratégia e-SUS $\mathrm{AB}$ propõe a informatização da Atenção Básica à Saúde por meio de dois sistemas de softwares, sendo eles o Cadastro de Dados Simplificado (CDS) e o Prontuário Eletrônico do Cidadão (PEC), objetivando reestruturar o registro das informações em todo o território nacional. ${ }^{(3)}$

A estratégia e-SUS AB acarreta alterações estruturais no conjunto de ideias e na prática dos profissionais e usuários dos serviços de saúde, caracterizando-se como uma inovação. A inovação é gerada quando toda e qualquer ideia, prática ou objetivo é notado como "novo" por um indivíduo ou conjunto, o que acontece no período da implantação da inovação, sendo fundamental o processo de aceite ou não da inovação pelo indivíduo. ${ }^{(4)}$

A adoção de uma inovação provém diretamente do modo como ela é demonstrada a seus futuros adotantes, podendo ser aceita ou rejeitada. (4) É necessário considerar que a estratégia e-SUS AB está em fase de implantação em todo o território nacional necessitando ser avaliada. ${ }^{(3,5)}$ Desta feita, buscou-se analisar a difusão da inovação tecnológica "estratégia e-SUS AB" em uma equipe de saúde da família do oeste de Minas Gerais.

\section{METODOLOGIA}

Trata-se de um estudo de caso único, holístico, de abordagem qualitativa. A escolha pelo estudo de caso se justifica por permitir a investigação de um fenômeno social complexo, que mantém as características holísticas e significativas dos acontecimentos da vida real, como os processos organizacionais e administrativos. ${ }^{(6)}$

Neste sentido, a estratégia e-SUS AB corresponde a um fenômeno complexo de processos organizacionais e administrativos, pela fase inicial de implantação e utilização de seus sistemas de softwares. Assim, definiu-se como unidade de análise a difusão da inovação estratégia e-SUS AB.

Como referencial teórico utilizou-se a Teoria da Difusão da Inovação, em que a difusão é concebida como um tipo especial de comunicação, no qual uma inovação/mudança seja o tema central. A difusão de uma inovação compreende o processo em que uma inovação é comunicada através de certos canais de comunicação, por um período, entre os membros de um sistema social. ${ }^{(4)}$

Em caráter complementar, também utilizamos o Modelo de Aceitação de Tecnologias ${ }^{(8)}$, quando se acredita que a aceitação de uma tecnologia está atrelada à percepção do sujeito sob duas dimensões fundamentais, que são a facilidade de uso percebida e a utilidade percebida. O Quadro 1 detalha os modelos teóricos, as dimensões, os elementos e atributos da Teoria da Difusão da Inovação e do Modelo de Aceitação de Tecnologias, além das fontes de evidências utilizadas. 
Quadro 1 - Fontes de evidências à luz das lentes teóricas da Teoria da Difusão da Inovação e do Modelo de Aceitação de Tecnologias, município de pequeno porte, Região de Saúde Oeste de Saúde, Minas Gerais, Brasil, 2016

\begin{tabular}{|c|c|c|}
\hline Dimensões & Atributos & Fontes de evidência \\
\hline \multicolumn{3}{|c|}{ TEORIA DA DIFUSÃO DA INOVAÇÃO (TDI) } \\
\hline \multirow{5}{*}{$\begin{array}{l}\text { Elemento } \\
\text { Inovação }\end{array}$} & Vantagem Relativa: Percepção de que o uso da inovação é vantajoso. & Entrevista \\
\hline & $\begin{array}{l}\text { Compatibilidade: Inovação compatível com os valores, experiências e } \\
\text { crenças praticadas. }\end{array}$ & $\begin{array}{l}\text { Entrevista } \\
\text { Observação }\end{array}$ \\
\hline & $\begin{array}{l}\text { Complexidade: Percepções sobre a dificuldade em utilizar a } \\
\text { inovação. }\end{array}$ & $\begin{array}{l}\text { Entrevista } \\
\text { Observação }\end{array}$ \\
\hline & Experimentação: Período de teste da inovação. & Entrevista \\
\hline & Observabilidade: Percepção dos resultados obtidos através da inovação. & Entrevista Observação \\
\hline $\begin{array}{l}\text { Canais de } \\
\text { Comunicação }\end{array}$ & $\begin{array}{l}\text { Meios em que as mensagens circulam entre os indivíduos, considerando a } \\
\text { natureza da troca de mensagens. }\end{array}$ & Entrevista Observação \\
\hline Tempo & $\begin{array}{l}\text { A dimensão do tempo entre o contato com a inovação e a decisão de } \\
\text { aceitar ou rejeitar. }\end{array}$ & Entrevista \\
\hline Sistema Social & $\begin{array}{l}\text { Membros ou unidades de um sistema social podem ser individuais, } \\
\text { grupos informais ou organizações. }\end{array}$ & $\begin{array}{l}\text { Entrevista } \\
\text { Observação }\end{array}$ \\
\hline \multicolumn{3}{|c|}{ MODELO DE ACEITAÇÃO DE TECNOLOGIAS } \\
\hline $\begin{array}{l}\text { Facilidade de } \\
\text { uso percebida }\end{array}$ & Percepção de que o uso da tecnologia não demanda esforço ou dificuldade. & Entrevista Observação \\
\hline $\begin{array}{l}\text { Utilidade } \\
\text { percebida }\end{array}$ & Percepção de que o uso da tecnologia melhora o desempenho. & Entrevista Observação \\
\hline
\end{tabular}

Definiu-se como cenário de estudo um município localizado na região Oeste de Minas Gerais, com uma população de 46.482 habitantes, com 100\% de cobertura populacional pela Estratégia Saúde da Família. A escolha do referido município justificou-se pela necessidade de analisar a difusão da inovação em um município de pequeno porte (< 50.000 habitantes), em processo inicial de implantação da estratégia e-SUS AB e por ser de fácil acesso aos pesquisadores.

Uma equipe de saúde da família do município foi selecionada por meio de sorteio aleatório. Todos os 14 possíveis participantes da referida equipe foram incluídos. O período da coleta de dados aconteceu entre os meses de março e julho de 2016, sendo os participantes codificados com a letra E, seguida do número sequencial da entrevista como E01.

Para a coleta de dados, utilizou-se a entrevista aberta e a observação direta orientada por um roteiro de observação e registrada em um diário de campo como Notas de Observação (NO). As entrevistas duraram em média 50 minutos e a observação foi realizada em cinco dias de uma semana típica de trabalho. O Quadro 2 descreve as questões e itens de observação presentes nos instrumentos de coleta de dados.

Quadro 2 - Perguntas de pesquisa e aspectos observados na coleta de dados, de acordo com a Teoria da Difusão da Inovação e o Modelo de Aceitação de Tecnologias, município de pequeno porte, Região de Saúde Oeste, Minas Gerais, Brasil, 2016. (continua)

\begin{tabular}{|c|c|}
\hline Dimensões & Questões/Itens de observação \\
\hline $\begin{array}{c}\text { Inovação } \\
\text { Observabilidade } \\
\text { Utilidade } \\
\text { Percebida }\end{array}$ & $\begin{array}{l}\text { Fale-me sobre e-SUS AB em sua unidade de saúde da família? } \\
\text { Influências observáveis sobre o trabalho. } \\
\text { Benefícios do uso do e-SUS AB. } \\
\text { Utilidade do software no processo de trabalho. } \\
\text { Presença de computadores nas estações de trabalho. } \\
\text { Utilização/presença de formulários, cadernos ou outros meios de registro em papel alheios ao } \\
\text { e-SUS AB. }\end{array}$ \\
\hline
\end{tabular}




\begin{tabular}{|c|c|}
\hline Canais de & $\begin{array}{l}\text { Fale-me sobre as fontes de informações que você teve ou tem acesso sobre o e-SUS AB. } \\
\text { Canais de comunicação sobre o e-SUS AB }\end{array}$ \\
\hline Comunicação & $\begin{array}{l}\text { Presença ou conhecimento dos Manuais disponibilizados pelo Ministério da Saúde. } \\
\text { Informativos e registros das capacitações já realizadas. }\end{array}$ \\
\hline $\begin{array}{l}\text { Tempo } \\
\text { Experimentação }\end{array}$ & $\begin{array}{l}\text { Descreva como tem sido o processo de implantação do e-SUS AB em sua unidade desde o início } \\
\text { até o presente momento. }\end{array}$ \\
\hline & Como você avalia o e-SUS AB em relação ao sistema que você utilizava anteriormente? \\
\hline Vantagem & rotina de tr \\
\hline Relativa & $\begin{array}{l}\text { Volume de documentos e formulários em uso nas estações de trabalho (recepção, consultórios e } \\
\text { salas de procedimento). }\end{array}$ \\
\hline Compatibilidade & $\begin{array}{l}\text { Fale-me sobre a adequação do e-SUS AB às suas necessidades de trabalho. } \\
\text { Atendimento às necessidades dos profissionais } \\
\text { Estrutura física das unidades, bem como conexão de internet, computadores e impressoras } \\
\text { Escala de trabalho dos profissionais. }\end{array}$ \\
\hline Complexibilidade & $\begin{array}{l}\text { Fale-me sobre os instrumentos utilizados no e-SUS AB (fichas, software, manuais, etc.). } \\
\text { Dificuldades na utilização do sistema e demais instrumentos (fichas CDS/e-SUS AB). }\end{array}$ \\
\hline Facilida & Dificuldade ou facilidade dos profissionais para o uso do e-SUS AB \\
\hline Percebida & Dificuldade ou facilidade para o uso de computadores e impressoras. \\
\hline Sistema Social & $\begin{array}{l}\text { Formação de grupos e interação entre os profissionais, a partir do e-SUS AB. } \\
\text { Memorandos e ofícios das instâncias municipal, estadual ou federal. } \\
\text { Relações interpessoais. }\end{array}$ \\
\hline
\end{tabular}

Os dados foram sistematizados através do software Atlas ti e analisados por meio da Análise de Conteúdo modalidade Temático-Categorial. ${ }^{(9)}$ A análise dos dados permitiu recortar as unidades de registro e codificar, atendendo aos princípios da exaustividade, representatividade, homogeneidade e pertinência..$^{(9)}$

O estudo foi aprovado pelo Comitê de Ética em Pesquisa da Universidade Federal de São João Del Rei, sob parecer do n.ำ 1.247.996/2015.

\section{RESULTADOS}

O estudo contou com 14 participações, sendo um médico, uma enfermeira, um dentista, dois técnicos de enfermagem, dois técnicos de saúde bucal, cinco agentes comunitários de saúde, um auxiliar administrativo e um nutricionista. Verificou-se que a maioria dos participantes era do sexo feminino, com faixa etária variando entre 34 e 51 anos, em média 42 anos.

Em relação ao tempo de serviço, médico e enfermeira apresentaram maior tempo de experiência com, respectivamente, 15 e 12 anos. Considerando toda a equipe, a média de tempo de serviço foi de seis anos. Do total de participantes, sete possuíam o nível médio de escolaridade, três eram graduados e quatro possuíam alguma especialização lato sensu. Dentre os participantes, cinco eram servidores públicos concursados na prefeitura municipal e com média de tempo de serviço de cinco anos.

\section{A inovação e-SUS AB e seus atributos}

Nesta primeira categoria, em relação ao atributo vantagem relativa, os participantes teceram comparações entre sistemas anteriores, utilizados pelo município, e o e-SUS AB.

[...] O e-SUS é mais completo. [...] Antes o SIAB não tinha tanta coisa para passar, era só idade, data de nascimento, não pedia cartão SUS, não pedia nome da mãe. Esse pede DN, cartão SUS, CPF, RG, endereço [...]. (E2)

Porém, alguns instrumentos de coleta de sistemas anteriores ainda são utilizados, pois tais dados não estão presentes nos sistemas de software da estratégia e-SUS AB. A cena observada destaca esta situação. 
As agentes de saúde carregavam consigo pranchetas com formulários impressos do e-SUS, além da ficha A do extinto SIAB. Para a rotina diária de trabalho das ACSs, as fichas do e-SUS apresentam-se como uma atividade a mais. (NO)

Alguns participantes perceberam melhoras em alguns processos no cotidiano de trabalho em relação ao sistema anterior.

[...] As fichas que eram feitas manuais, aonde eu cheguei a fazer fechamento do mês, quando era com o SIAB, contando quadradinho por quadradinho, de consultas médicas, de datas e tudo. Quando não tinha tempo de fechar no dia, a gente deixava para fechar no final do mês [...]. (E5)

Os participantes também verbalizaram sobre as incompatibilidades da inovação em relação à infraestrutura disponibilizada e ao processo de trabalho da equipe de saúde da família.

[...] Às vezes, o sistema cai muito, demora, fica muito tempo ali, na frente do computador, esperando. Tem uns horários que é mais difícil. No final do mês, às vezes, acontece da internet estar mais devagar e atrasa nosso serviço na unidade [...]. (E4)

A baixa conectividade e o deficiente número de computadores também foram lembrados como incompatíveis com as demandas de trabalho.

[...] Imagina, estou aqui atendendo, como técnica, aferindo a sua pressão, fazendo o meu serviço, vim aqui colocar no computador, estava prontinho e cairia lá na sala do médico. Ficaria tudo direitinho, iria ser muito bom se funcionasse, se o doutor tivesse computador, pois lá não tem, são poucos computadores na unidade. [...]. (E3)

Em relação ao atributo complexidade, reconheceu-se a falta de conhecimento sobre como operacionalizar a inovação para extrair informações e a incompreensão acerca de alguns códigos disponibilizados.

[...] não entendo aqueles códigos de procedimentos [...] tem um monte de linguagem de programação lá no e-SUS que eu não entendo. Códigos de diagnóstico, e de outras coisas que ainda não consegui entender, e ninguém me explicou [...]. (E6)

[...] precisava de um relatório de hipertensos, diabéticos e eu não consigo [...] Pois até então eles não conseguiram nos passar como extrair do sistema estes relatórios. Como que você vai se programar e agendar?[...]. (E8)

A experimentação da inovação foi um atributo percebido pelos participantes e esteve atrelada às mudanças súbitas e constantes nos instrumentos de coleta de dados.

[...] com a mudança das fichas, foi um susto muito grande porque aí veio tudo muito rápido e um monte de ficha, mudando as fichas, cada dia uma ficha nova, quando a gente pensou que sabia tudo da ficha, aparecia outra [...]. (E14)

Em relação ao atributo observabilidade, alguns participantes perceberam efeitos positivos da inovação, como o registro da produção, etapa fundamental para o repasse de recursos financeiros ao município. Outra aplicabilidade do software é para o acompanhamento de grupos prioritários:

[...] no e-SUS, tem como ver [...] quantas consultas eu fiz, quantos grupos eu fiz, quantas pessoas que eu atendi. Isso é importante [...] bater metas para o repasse financeiro [...] consigo acompanhar e planejar melhor a assistência [...]. (E6)

Por outro lado, outros entrevistados traduzem estes efeitos como influências negativas da inovação sobre a assistência, devido à sobrecarga administrativa gerada pela inovação tecnológica, além do retrabalho.

[...] trabalho meio que perdida [...] sobrecarrego a minha parte administrativa com o sistema e estou deixando muito a assistência a desejar [...] o médico não digita [...] como que eu delego uma digitação de uma ficha que leva CID? [...]. (E12)

Na unidade, foi possível observar um grande número de impressos relacionado à estratégia e-SUS $A B$, acumulados para posterior digitação. (NO) 


\section{Os canais de comunicação envolvidos na difusão da inovação}

As pessoas foram lembradas como principais canais de comunicação contribuindo para a difusão da inovação, principalmente a partir do desenvolvimento de habilidades em relação à tecnologia, que ocorre a partir da interação entre profissionais no cotidiano de trabalho.

[...] Aqui um ensinou para o outro, um aprendeu e foi ensinando para os outros [...] temos muitas dúvidas ainda [...]. (E7)

Para sanar as dúvidas e auxiliar em momentos em que o sistema apresenta problemas, a figura do técnico de informática se sobressaiu nas falas dos profissionais:

[...] a gente ligava para o técnico ou ele vinha e orientava a gente. Ensinou a gente a trabalhar e a mexer. Se hoje em dia o sistema travar, a gente tem que ligar pra ele e ele tem que vir aqui para poder $\operatorname{ver}[\ldots] .(\mathrm{E} 14)$

Por outro lado, os manuais de operacionalização do sistema são desconhecidos ou não estão disponíveis para os profissionais consultarem em caso de dúvidas:

[...] Não há manuais disponibilizados, desconheço. A ajuda que a gente tem é uma ajudando a outra e o técnico de informática [...]. (E10)

\section{Influências do sistema social e do tempo na difusão da inovação}

Os profissionais relataram que o processo de implantação da inovação se deu dentro de um período curto de tempo, após a troca do gestor municipal.

[...] chegou assim, de repente. Entrou prefeito novo e já mudou, a gente já foi fazendo os cadastros pelo computador [...]. (E1)

Em outro relato foi especificado o tempo e parte das condições de implantação

[...] acho que uns 40 a 50 dias. Não foi muito tempo, pois logo fizeram o treinamento e mandaram as coisas, sem qualquer estrutura [...]. (E11)

Além do curto espaço de tempo para implantação, os profissionais ressaltaram que o processo de implantação da inovação tem acontecido de forma impositiva, a partir de instâncias superiores, e isso tem contribuído para gerar deficiências em tal processo.

[...] a gente sabe que tudo que vem de cima para baixo ela vai se perdendo, as coisas, então vai chegando, e quando chega para a gente, já chega com algumas deficiências [...]. (E8)

Outro entrevistado destacou a maneira como a implantação vem acontecendo, de forma súbita, não sistematizada e a partir de pressões.

[...] a implantação está acontecendo a toque de caixa. O que eu sei te falar é o seguinte: veio uma ordem, parece que de Brasília, que tinha que implantar o e-SUS e aí a secretaria ficou muito apertada, atribulada e convocou todos os profissionais médico e enfermeiro para fazer um treinamento para o e-SUS [...]. (E11)

Em uma cena observada foi possível verificar que alguns profissionais estão resistentes à implantação, mas são movidos pela imposição de instâncias superiores. Sentem-se inseguros com a situação e apresentam muitas dúvidas.

Observo uma grande resistência por parte dos profissionais para implantar o e-SUS. Percebo insegurança e medo decorrente das dúvidas relacionadas ao software e seu uso. (NO)

O Quadro 3 a seguir sintetiza os elementos/atributo se as percepções sobre a facilidade de uso e utilidade, potencializando a aceitação ou rejeição da inovação e-SUS AB em seu processo de difusão, verificados neste estudo. 
Quadro 3 - Percepção dos profissionais de saúde da Atenção Básica sobre elementos que potencializam a rejeição e a aceitação da inovação e-SUS AB, município de pequeno porte, Região de Saúde Oeste, Minas Gerais, Brasil, 2016

\begin{tabular}{|c|c|c|c|}
\hline & $\begin{array}{l}\text { Elementos/ } \\
\text { Atributos }\end{array}$ & $\begin{array}{l}\text { Percepções sobre a facilidade de uso e } \\
\text { utilidade potencializando a rejeição do } \\
\text { e-SUSAB }\end{array}$ & $\begin{array}{l}\text { Percepções sobre a facilidade de } \\
\text { uso e utilidade potencializando } \\
\text { a aceitação do e-SUSAB }\end{array}$ \\
\hline \multirow{5}{*}{ 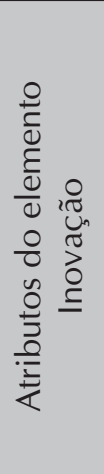 } & Complexibilidade & $\begin{array}{l}\text { Dificuldade para extrair relatórios } \\
\text { Incompreensão acerca de alguns códigos }\end{array}$ & - \\
\hline & Observabilidade & $\begin{array}{l}\text { Sobrecarga administrativa gerada pela } \\
\text { inovação tecnológica, além do retrabalho }\end{array}$ & \multirow[t]{2}{*}{$\begin{array}{l}\text { Suporte do sistema no } \\
\text { planejamento da assistência }\end{array}$} \\
\hline & Compatibilidade & $\begin{array}{l}\text { Baixa conectividade e o baixo número de } \\
\text { computadores }\end{array}$ & \\
\hline & Vantagem Relativa & $\begin{array}{l}\text { Alguns instrumentos de coleta de sistemas } \\
\text { anteriores ainda são utilizados }\end{array}$ & \multirow[t]{2}{*}{$\begin{array}{l}\text { Dados mais completos } \\
\text { Melhoras de alguns processos }\end{array}$} \\
\hline & Experimentação & $\begin{array}{l}\text { Mudanças súbitas e constantes nos } \\
\text { instrumentos de coleta de dados }\end{array}$ & \\
\hline & Sistema Social & $\begin{array}{l}\text { Pressões de instancias superiores para a } \\
\text { implantação }\end{array}$ & \multirow[b]{2}{*}{$\begin{array}{l}\text { As pessoas como canais de } \\
\text { comunicação. }\end{array}$} \\
\hline Canai & ais de Comunicação & $\begin{array}{l}\text { Desconhecimento de manuais sobre a } \\
\text { inovação }\end{array}$ & \\
\hline & Tempo & Implantação repentina e não sistematizada & - \\
\hline
\end{tabular}

\section{DISCUSSÃO}

A implantação da estratégia e-SUS AB tem enfrentado situações que potencializam, predominantemente, a sua rejeição. Verificamos a necessidade de reorganizações administrativas do trabalho, a necessidade de recadastramento da população e o preenchimento manual de diversas fichas. Reconheceu-se a dificuldade de extrair relatórios e incompreensões acerca dos códigos utilizados nos sistemas de software. Além disso, as mudanças súbitas e constantes nos instrumentos utilizados para a coleta de dados dificultaram o acesso à informação.

O cenário gera sobrecarga e retrabalho no cotidiano dos profissionais, elementos que tendem a fragilizar a aceitação de uma inovação tecnológica. ${ }^{(4,8)}$ Neste contexto, a estratégia e-SUS AB, apesar de ser reconhecida como uma inovação pelos pesquisados, torna-se incompatível com as demandas de um processo de trabalho estabelecido a priori.

Assim, a incompatibilidade e a complexidade atribuída à inovação acabam por desenvolver nos profissionais a percepção de que a tecnologia em implantação é um instrumento do processo de trabalho, que, somado aos demais, representa uma sobrecarga com repercussões negativas sobre a assistência. Assim, a percepção da utilidade da inovação para o processo de trabalho e sua facilidade de uso é prejudicada. ${ }^{(8)}$

Em estudos nacionais e internacionais a incompatibilidade de sistemas de informação em relação ao processo de trabalho em saúde, bem como a sua complexidade de uso foram fatores que contribuíram para o seu insucesso e com repercussões negativas sobre a gestão e assistência. ${ }^{(1-2,5,10-11)}$

O desenvolvimento de tecnologias da informação deve ser adequado às necessidades informacionais de seus potenciais usuários, além de contribuir para a gestão das informações que emergem do processo de trabalho. ${ }^{(12-13)}$ Para ser amplamente adotada como uma tecnologia inovadora,deve instrumentalizar as pessoas com vistas a qualificar o fluxo de informações, o planejamento e a tomada de decisões assertiva, pois do contrário tende a ser rejeitada. ${ }^{(4,8)}$ 
Tal rejeição pode ser caracterizada como a sua não utilização pelos profissionais, tornando-se uma tecnologia obsoleta. Mas também pode ser caracterizada por sua subutilização, em que a tecnologia não é utilizada adequadamente pelos profissionais, gerando informações infidedignas e com pouca aplicabilidade no processo de trabalho. ${ }^{(14)}$

Vários estudos têm demonstrado a subutilização de sistemas de informação para fins de planejamento, principalmente, o planejamento local. ${ }^{(2,12-14)}$ Além disso, a gestão do cuidado também tem sido prejudicada quando da utilização inadequada de sistemas de informação, acarretando impactos negativos sobre a segurança do paciente, famílias e comunidades. ${ }^{(11,15)}$

A subutilização não pode ser repetida com os sistemas de software da estratégia e-SUS AB, pois a sua principal premissa é proporcionar a gestão da informação voltada para o planejamento e a tomada de decisões locais. ${ }^{(3,5)}$ Espera-se que a utilização dos sistemas de software da estratégia e-SUS AB favoreçam a sistematização de dados, desde a coleta até a análise das informações, instrumentalizando a organização do trabalho e a gestão do cuidado. ${ }^{(3,5)}$ Entretanto, para que isso de fato aconteça, é preciso institucionalizar a inovação no sentido de sua utilização maximizada como um elemento crucial do processo de trabalho.

A institucionalização necessária não é aquela apenas normatizada, pois isso já se faz presente no caso da estratégia e-SUS AB. ${ }^{(3)}$ É preciso ir além: a inovação tecnológica precisa ser reconhecida pelos profissionais como necessária, útil e aplicável ao processo de trabalho característico da atenção básica à saúde. A utilidade do software deve ser compartilhada pelos profissionais, evidenciando a sua utilização nas atividades cotidianas como parte do processo de trabalho.

Em países onde a informatização vem acontecendo no contexto da atenção primária à saúde, a utilização de tecnologias da informação tem favorecido o processo de trabalho e a aceitação entre os profissionais de saúde. ${ }^{(16-17)}$

Assim, as pessoas precisam ser consideradas na difusão de inovações. Sobretudo, há a necessidade de desenvolver significados que representem percepções positivas sobre a inovação entre os indivíduos. $(4,8,10,15)$ Neste sentido, é preciso envolver os profissionais de saúde no processo de implantação da estratégia e-SUS AB.

Nos achados deste estudo, evidenciou-se o desconhecimento dos participantes sobre os principais meios de comunicação da inovação, disponibilizados pelo governo, para auxiliar a implantação da inovação. Assim, é preciso ampliar e diversificar os canais de comunicação, além de torná-las acessíveis. $(3,4,8)$

O preparo e o planejamento que antecedem o processo de difusão da inovação são de fundamental importância por significar como os potenciais usuários tomarão conhecimento da inovação, dando início ao processo de difusão e adoção. ${ }^{(4,15)}$ Entretanto, no cenário estudado, há a percepção de que o processo de implantação da estratégia e-SUS AB tem sido repentino e verticalizado.

As pessoas representam fator sensível de sucesso na implantação e adoção de tecnologias da informação em saúde. ${ }^{(10,15,18)}$ Alguns estudos demonstram que o envolvimento de profissionais de saúde no desenvolvimento e avaliação de tecnologias, além de sua capacitação para a utilização, contribuem para a obtenção de significados favoráveis para a adoção da tecnologia. ${ }^{(10,15,18)}$ Tais estudos até sugerem a presença de profissionais de saúde nas equipes de tecnologia da informação, responsáveis pela implantação. O envolvimento das pessoas no processo de implantação de inovações tecnológicas, no contexto da saúde, também contribui para a redução de resistências. ${ }^{(10,15,18)}$

A difusão da inovação e-SUS AB também foi percebida pelos pesquisados como uma exigência dos gestores de instâncias superiores, vinculada a repasses financeiros, reforçando o seu reconhecimento como um elemento discrepante do processo de trabalho. Neste sentido, a utilização dos sistemas de software é reduzida à necessidade de cumprir os requisitos de alimentação de dados que serão enviados às esferas municipal, estadual e federal, portanto, não há a necessidade de discuti-los, analisá-los ou utilizá-los. Esta situação tende a influenciar diretamente o modo como a tecnologia é percebida e utilizada, além da fidedignidade dos dados produzidos. ${ }^{(2,12-14)}$ 
Neste contexto, os dados que alimentam os sistemas de software podem ser subnotificados ou supranotificados a partir do critério de metas estabelecidas a priori, mas sem representar, de fato, a veracidade dos fatos. Estes achados já foram verificados em outros estudos nacionais relacionados a sistemas de informação em saúde. ${ }^{(2,14,19)}$ É preciso romper com este modelo informacional no que tange aos sistemas de informação de abrangência nacional, já constatado em outros estudos, nos quais os profissionais de saúde fazem coletas de dados sem sentido, voltadas a alimentar as esferas de gestão, centralizando informações e dificultando a tomada de decisões locais. ${ }^{(1,14,20)}$

A estratégia e-SUS AB emerge desta necessidade, de integrar as informações dos pontos da rede assistencial de saúde, mas também da necessidade de se fazer a gestão das informações voltadas para a transformação da realidade local. ${ }^{(3,21-22)}$

Assim, profissionais de saúde e gestores de todos os pontos da rede assistencial precisam ser estimulados e qualificados para utilizar as tecnologias disponíveis, no sentido de organizar o trabalho e gerenciar os serviços locais, não apenas para enviar dados para instâncias superiores, visando o repasse de verbas. Isso diminui significativamente o potencial de transformação da realidade que as inovações tecnológicas podem proporcionar. ${ }^{(14,20,23)}$

O estudo apresenta como limite a sua capacidade de generalização, porém seus resultados podem direcionar outras investigações que aprofundem a compreensão sobre a difusão de tecnologias em serviços de saúde e suas repercussões sobre as relações entre os profissionais e pacientes.

\section{- CONCLUSÃO}

Neste estudo, propusemos analisar a difusão da inovação estratégia e-SUS AB em uma equipe de saúde da família de um município do oeste mineiro. Verificamos que a difusão da estratégia e-SUS $A B$, como uma inovação tecnológica, tem apresentado situações que potencializam a sua aceitação, mas, predominantemente, situações que se tornam desafiadoras, pois potencializam a sua rejeição. Tais situações precisam ser suplantadas com vistas a qualificar e efetivar a difusão da inovação tecnológica.

Acredita-se que outras investigações podem ser delineadas para analisar a difusão do prontuário eletrônico do cidadão da estratégia e-SUS $A B$, suas influências sobre o cotidiano de trabalho, sua aceitação pelos profissionais, seus efeitos sobre a segurança dos pacientes, sobre a gestão do cuidado e sobre o processo de trabalho.

\section{- AGRADECIMENTO}

Esta pesquisa foi financiada pela Fundação de Amparo à Pesquisa do Estado de Minas Gerais (FAPEMIG), através do edital APQ-00337-15, e pelo Conselho Nacional de Desenvolvimento Científico e Tecnológico, através do edital CNPQ - Processo 404653/2016-2.

\section{REFERÊNCIAS}

1. Cavalcante RB, Pinheiro MMK, Watanabe YJÁ, Silva CJ. Grupo técnico de informação em saúde e populações: contribuições para a política nacional de informação e informática em saúde. Perspect. ciênc. inf.[Internet] 2015; 20(1) [acesso em 17 fev de 2017]. Disponível: http://dx.doi.org/10.1590/1981-5344/1905.

2. Carreno I, Moreschi C, Marina B, Hendges DJB, Rempel C. de Oliveira MMC. Análise da utilização das informações do Sistema de informação de Atenção Básica (SIAB): uma revisão integrativa. Ciênc. saúde coletiva. [Internet] 2015;20(3) [acesso em 11 out. 2017]. Disponível: http://dx.doi.org/10.1590/1413-81232015203.17002013. 
3. Ministério da Saúde (BR). e-SUS Atenção Básica: manual de implantação. [Internet] Brasília: Ministério da Saúde; 2014 [acesso em 29 dez 2015]. Disponível: http://189.28.128.100/dab/docs/portaldab/documentos/manual_implantacao_ esus.pdf.

4. Rogers EM. Diffusion of innovation. 3eed. New York: The Free Press; 2003.

5. de Oliveira AEC, de Lima IMB, do Nascimento JA, Coelho HFC, dos Santos SR. Implantação do e-SUS AB no Distrito Sanitário IV de João Pessoa (PB): relato de experiência. Saúde debate. [Internet] 2016;40(109) [acesso em 10 out. 2016]. Disponível: http://dx.doi.org/10.1590/0103-1104201610917.

6. Yin R. Estudo de caso: planejamento e métodos. 5ª ed. Porto Alegre: Bookman; 2015.

7. Minayo MCS. Análise qualitativa: teoria, passos e fidedignidade. Ciênc. saúde coletiva. [Internet] 2012; 17(3) [acesso em 11 out 2016]. Disponível: http://dx.doi.org/10.1590/S1413-81232012000300007.

8. Davis FD. Perceived usefulness, perceived ease of use, and user acceptance of information technology.MIS Quarterly [Internet] 1989;13(3) [acesso em 12 out 2016]. Disponível: http://www.jstor.org/stable/249008.

9. Bardin L. Análise de conteúdo. 4ª ed. Lisboa: 70; 2015.

10. Cucciniello M, Lapsley I, Nasi G, Pagliari C. Understanding key factors affecting electronic medical record implementation: a sociotechnical approach. BMC Health Services Research.[Internet] 2015;15(268) [acesso em 15 abr 2017].Disponível: http://dx.doi.org/10.1186/s12913-015-0928-7

11. Austyn S, Hildegard K. Two years of unintended consequences: introducing an electronic health record system in a hospice in Scotland. J Clin. Nurs. [Internet] 2016; 26(9) [acesso em 21 out. 2016]. Disponível: http://dx.doi.org/10.1111/ jocn.13576.

12. Holmes ES, dos Santos SR, Almeida AF, de Oliveira JHD, de Carvalho GDA, da Fonseca LCT, et al. Health information systems in the decision-making process in primary care. International Archives of Medicine Section: Global Health \& Health Policy. [Internet] 2016; 9(2) [acesso em 10 dez 2016]. Disponível: http://dx.doi.org/10.3823/1873.

13. Martins LMP, Silva EM, Marques D. Informação em saúde na ótica de enfermeiras de saúde da família. Rev. Min. Enferm (REME). [Internet] 2016; 20 [acesso em 10 nov 2016]. Disponível: http://dx.doi.org/10.5935/1415-2762.20160002.

14. Cavalcante RB, Bernardes MFVG, Gontijo TL, Guimarães EAA, Oliveira VC. Sistema de informação da atenção básica: potencialidades e subutilização no processo decisório. Cogitare enferm. [Internet] 2013; 18(3)[acesso em 09 mar 2018]. Disponível: http://dx.doi.org/10.5380/ce.v18i3.33555.

15. Meeks DW, Takian A, Sittig DF, Singh H, Baber N. Exploring the sociotechnical intersection of patient safety and electronic health record implementation. J. Am. Med. Inform. Assoc. [Internet] 2014; 21(1) [acesso em 03 mai 2017]. Disponível: http://dx.doi.org/10.1136/amiajnl-2013-001762.

16. Rittenhouse DR, Ramsay PP, Casalino LP, McClellan S, Kandel ZK, Shortell S M.Increased Health Information Technology Adoption and Use Among Small Primary Care Physician Practices Over Time: A National Cohort Study. Ann. Fam. Med. [Internet] 2017; 15 (1) [acesso em 05 mar 2018]. Disponível: https://doi.org/10.1370/afm.1992.

17. Anderson D, Zlateva I, Khatri K, Ciaburri N. Using Health Information Technology to Improve Adherence to Opioid Prescribing Guidelines in Primary Care. Clin. J. Pain. [Internet] 2015; 31 (6) [acesso em 05 mar 2018]. Disponível: https://doi.org/10.1097/AJP.0000000000000177.

18. Zakaria N,Mohd Y, Shafiz A.Understanding Technology and People Issues in Hospital Information System (HIS) Adoption: Case study of a tertiary hospital in Malaysia.J. Infect Public Health. [Internet] 2016; 9 (6) [acesso em 17 abr 2017].Disponível: https://doi.org/10.1016/j.jiph.2016.08.017.

19. Wendt CJK, de Lima SBS. Sistema de informação da atenção básica no contexto da saúde da família. Rev. Enferm. [Internet] 2015; 11(11) [acesso em 17 abr 2017]. Disponível:http://revistas.fw.uri.br/index.php/ revistadeenfermagem/article/view/1739/1976. 
20. Cavalcante RB, Kerr-Pinheiro MM, Guimarães EAA, Miranda RM. An overview of the definition and implementation of the Brazililian National Policy ond Health Data and Information Technology. Cad. Saúde Pública. [Internet] 2015; 31(5) [acesso em 03jan 2017]. Disponível: http://dx.doi.org/10.1590/0102-311X00095014.

21. Fornazin M, Joia LA. Remontando a rede de atores na implantação de um sistema de informação em saúde. Rev. adm. empres. [Internet] 2015; 55(5)[acesso em 17 fev2017]. Disponível: http://dx.doi.org/10.1590/S0034-759020150505.

22. Ministério da Saúde. Portaria n. 1.412, de 10 de julho de 2013. Institui o Sistema de Informação para a Atenção Básica (SISAB). Diário Oficial da União, 10 jul 2013.

23. Nodari $\mathrm{CH}$, Camargo ME, Olea PM, Dorion ECH, Claus SM. Configuração das práticas de inovação na atenção primária à saúde: estudo de caso. Ciênc. saúde coletiva. [Internet] 2015; 20(10) [acesso em 20 out 2016]. Disponível: http://dx.doi.org/10.1590/1413-812320152010.03262015. 